Current enhancement and negative differential conductance in parallel quantum dot systems

B. Tanatar, and V. Moldoveanu

Citation: AIP Conference Proceedings 1399, 287 (2011);

View online: https://doi.org/10.1063/1.3666366

View Table of Contents: http://aip.scitation.org/toc/apc/1399/1

Published by the American Institute of Physics 


\title{
Current enhancement and negative differential conductance in parallel quantum dot systems
}

\author{
B. Tanatar* and V. Moldoveanu ${ }^{\dagger}$ \\ *Department of Physics, Bilkent University, Bilkent, 06800 Ankara, Turkey \\ ${ }^{\dagger}$ National Institute of Materials Physics, P.O. Box MG-7, 077125 Bucharest-Magurele, Romania
}

\begin{abstract}
.
We present calculations on the transport properties of a double quantum dot (DQD) capacitively coupled to another individually biased dot. The effects of the intradot and interdot Coulomb interaction are included within the random-phase approximation (RPA) implemented in the Keldysh formalism. We show that by increasing the bias on the nearby dot the inelastic Coulomb scattering modifies the current in the double dot. The sign of the current depends on the detuning of the double dot levels and intradot transitions lead to negative differential conductance. The enhancement of the current due to the energy quanta transferred from the strongly biased dot suggests a quantum ratchet or Coulomb drag mechanism.
\end{abstract}

Keywords: Negative differential conductance, ratchet effect, drag effect

PACS: 73.23.Hk, 85.35.Ds, 85.35.Be, 73.21.La

\section{INTRODUCTION AND FORMALISM}

Quantum dot systems are ideal candidates for studying Coulomb effects on nanoscale transport. In two recent experiments Gustavsson et al. [1] and Gasser et al. [2] investigated the electronic transitions induced in a double quantum dot by the absorption of photons or acoustic phonons from the environment whose properties are changed by currents passing through nearby quantum points contacts. Khrapai et al. [3] on the other hand, reported a ratchet effect. The system considered in this experiments is a double quantum dot coupled to two leads and placed in the vicinity of a quantum point contact (QPC) which is also subjected to a finite bias At weak interdot tunneling the levels of each dot are detuned by an asymmetry energy $\Delta$ such that the system is in the Coulomb blockade regime. The transport measurements show that electrons can pass through the double dot if the bias applied on the QPC insures an energy transfer $\Delta$ between the two subsystem, via inelastic scattering.

Motivated by these experiments we investigate the electronic transport in parallel quantum dot systems. The electronic transitions induced in quantum dot systems by nearby biased detectors were previously studied within the master equation approach in the context of continuous measurement of a closed qubits by a nearby QPC. $[4,5]$ The same approach was used by Ouyang et al. for open quantum dots.[6] In this work we used the Keldysh formalism and the RPA for the Coulomb interaction [7] where the inelastic scattering processes are naturally included. The Hamiltonian splits in a part describing noninteracting and disconnected systems (i.e. double quantum dot (QDQ), quantum dot (D), leads (L)) and a sec- ond term which includes the coupling to the leads and the Coulomb interaction:

$$
H(t)=H_{\mathrm{DQD}}+H_{\mathrm{D}}+H_{\mathrm{L}}+\chi(t)\left(H_{\mathrm{T}}^{\mathrm{DQD}}+H_{\mathrm{T}}^{\mathrm{D}}+H_{\mathrm{I}}\right) .
$$

The switching function $\chi(t)$ vanishes in the remote past and reaches a constant value in the long-time limit. The numerical simulations are performed for lattice Hamiltonians. The creation/annihilation operators are $c_{n_{i}}^{\dagger} / c_{n_{i}}$ where $n_{i}$ denotes the site $n$ of the $\mathrm{QD}_{i} . H_{\mathrm{DQD}}$ then reads $(\langle$,$\rangle denotes nearest neighbor summation):$

$$
H_{\mathrm{DQD}}=\sum_{i} \sum_{n \in Q D_{i}}\left(\varepsilon_{n}+V_{i}\right) c_{n}^{\dagger} c_{n}+\sum_{\langle m, n\rangle}\left(t_{m n} c_{n}^{\dagger} c_{n}+h . c\right)
$$

where $V_{i}$ is a constant added to the onsite energies $\varepsilon_{n}$ simulating a gate potential applied on $\mathrm{QD}_{\mathrm{i}}$ and $t_{m n}$ are hopping constants. By convention the sites $i=1,2$ belong to $\mathrm{QD}_{1}$ and $i=3,4$ to $\mathrm{QD}_{2}$. The other dot is also described as a 4-site one dimensional chain and is coupled to two leads $\left(L_{d}\right.$ and $\left.R_{d}\right)$. Each lead is characterised by its chemical potential, the two biases given by $V_{\mathrm{DQD}}=\mu_{L}-\mu_{R}$ and $V_{\mathrm{D}}=\mu_{L}^{d}-\mu_{R}^{d}$. The interacting part is as usual $H_{\mathrm{I}}=\sum_{i, j} \sum_{m, n} W_{0, n_{i} m_{j}} c_{n_{i}}^{\dagger} c_{n_{i}} c_{m_{j}}^{\dagger} c_{m_{j}}$ where $W_{0, n_{i} m_{j}}=U /\left|r_{n}^{(i)}-r_{m}^{(j)}\right|$ is the bare interaction potential depending on the strength parameter $U$ and on the distance between a pair of sites. The tunneling Hamiltonians have standard form and are omitted here. The steadystate current entering the double dot from the left lead is given by ( $t_{L}$ is the hopping energy of the leads):

$$
\begin{gathered}
J_{\mathrm{DQD}}=\frac{e}{h} \int_{-2 t_{L}}^{2 t_{L}} d E \operatorname{Tr}\left\{\Gamma_{L} G^{R} \Gamma_{R} G^{A}\left(f_{L}-f_{R}\right)\right. \\
\left.-\Gamma_{L} G^{R} \operatorname{Im}\left(\Sigma_{I}^{<}+2 f_{L} \Sigma_{I}^{R}\right) G^{A}\right\},
\end{gathered}
$$



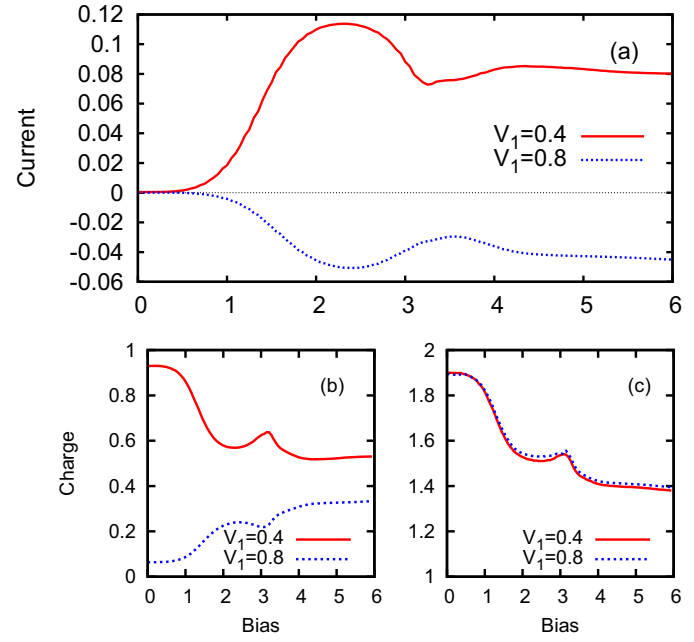

FIGURE 1. (Color online) (a) The current through the double dot as a function of the bias applied on the detector for two values of $V_{1}$. (b) and (c) The occupation numbers of the two dots for the same parameters as in (a). Other parameters: $U=0.15, \tau=0.1, v_{L}=v_{R}=0.35, V_{2}=-1.55$.

where the trace stands for the sum over the sites belonging to the double dot and $\Gamma^{L, R}$ are contact self-energies. The sign convention is such that $J_{\mathrm{DQD}}$ is positive if electrons flow from the left lead towards the double dot. The self-energies $\Sigma_{I}^{R}$ and $\Sigma_{I}^{<}$are calculated within the RPA scheme (the details are given in our previous work [7, 8]).

\section{RESULTS AND DISCUSSION}

For simplicity we consider identical quantum dots. The relevant parameters to be varied are the bias $V_{\mathrm{D}}$ applied to the strongly biased quantum dot and the two gate potentials $V_{1,2}$ applied to each dot which control the charge configuration in the double dot, that is the number of electrons in each dot. By varying $V_{1,2}$ one changes the energy detuning, defined as $\Delta=E_{m+1, n}-E_{m, n+1}$, where $E_{m, n}$ is the ground state configuration with $m$ electrons in $\mathrm{QD}_{1}$ and $n$ electrons in $\mathrm{QD}_{2}$. The hopping parameter $t_{S}$ in the DQD is chosen as energy unit and current is given in units of $e t_{S} / \hbar$. The interdot tunneling $\tau=0.1$.

Fig. 1(a) shows that by selecting different gate potentials applied on $\mathrm{QD}_{1}$ one can tune qualitatively different transport regimes. For $V_{1}=0.4$ the non-equilibrium fluctuations in the driving quantum dot induce a positive current in the $D Q D$, while for $V_{1}=0.8$ the current is negative, i.e. flows against the driving bias. In both cases a negative differential conductance regime is noticed in a certain bias range and the current eventually saturates at larger values of $V_{\mathrm{D}}$. The mechanism behind each regime is revealed by the behavior of the occupation numbers $N_{1}, N_{2}$ shown in Figs. 1(b) and (c). Due to the weak interdot coupling the charge in the second dot is less sensitive to the variation of $V_{1}$. For $V_{2}=0.4$ almost the same amount of charge is expelled from both dots in a similar way. The inelastic processes leading to the current are most likely the ones in which electrons tunnel from both dots to the leads. We see that for $V_{1}=0.8$ only a small amount of charge is localized on $\mathrm{QD}_{1}$, which means that its lowest level is now above the chemical potential of the leads. This also means that the detuning $E_{1,1}-E_{0,2}$ is positive and electrons inelastically tunnel from the highest level of $\mathrm{QD}_{2}$ to the lowest level of $\mathrm{QD}_{1}$. The scenario is confirmed by the numerical data, as $N_{1}$ increases while $N_{2}$ decreases. This negative ratchet current is entirely due to inelastic tunneling between the two dots. Electrons from $\mathrm{QD}_{2}$ can still tunnel from the highest level to the right lead but the level is more likely fed back from the same lead so a positive current is unlikely. In contrast, an electron that tunneled from $\mathrm{QD}_{2}$ to $\mathrm{QD}_{1}$ escapes more easily into the left lead because the weak interdot tunneling prevents relaxation in $\mathrm{QD}_{2}$. Note that the negative ratchet current emerges at a larger (threshold) value of $V_{\mathrm{D}}$ than the positive current, because in the latter case the minimal energy electrons need in order to escape into the drain lead is given by the difference between their energy and the chemical potential of the drain.

\section{ACKNOWLEDGMENTS}

B. T. acknowledges support from TUBITAK (108T743), TUBA, and EU-FP7 project UNAM-REGPOT (203953). This work is also supported in part by TUBITAK-BIDEP.

\section{REFERENCES}

1. S. Gustavsson, M. Studer, R. Leturcq, T. Ihn, K. Ensslin, D. C. Driscoll and A. C. Gossard, Phys. Rev. Lett. 99, 206804 (2007).

2. U. Gasser, S. Gustavsson, B. Küng, K. Ensslin, T. Ihn, D. C. Driscoll, and A. C. Gossard, Phys. Rev. B 79, 035503 (2009).

3. V. S. Khrapai, S. Ludwig, J. P. Kotthaus, H. P. Tranitz, and W. Wegscheider, Phys. Rev. Lett. 97, 176803 (2006)).

4. T. M. Stace and S. D. Barrett, Phys. Rev. Lett. 92, 136802 (2004).

5. B. Dong, N. J. M. Horing, and X. L. Lei, Phys. Rev. B 74, 033303 (2006).

6. S.-H. Ouyang, C.-H. Lam, and J. Q. You, Phys. Rev. B 81, 075301 (2010).

7. V. Moldoveanu and B. Tanatar, Phys. Rev. B 77, 195302 (2008).

8. V. Moldoveanu and B. Tanatar, Europhys. Lett. 86, 67004 (2009). 